\title{
BMJ Open Different exposure metrics of rotating night shift work and hyperhomocysteinaemia among Chinese steelworkers: a cross-sectional study
}

\author{
Shengkui Zhang (D , ${ }^{1}$ Yongbin Wang, ${ }^{2}$ Qinglin Li, ${ }^{1}$ Zhende Wang, ${ }^{1}$ Han Wang, ${ }^{1}$ \\ Chao Xue, ${ }^{1}$ Ying Zhu (D) ,' Weijun Guan, ${ }^{1}$ Juxiang Yuan ${ }^{1}$
}

To cite: Zhang S, Wang Y, Li Q, et al. Different exposure metrics of rotating night shift work and hyperhomocysteinaemia among Chinese steelworkers: a crosssectional study. BMJ Open 2020;10:e041576. doi:10.1136/ bmjopen-2020-041576

- Prepublication history and additional material for this paper is available online. To view these files, please visit the journal online (http://dx.doi.org/10. 1136/bmjopen-2020-041576).

Received 13 June 2020 Revised 07 0ctober 2020 Accepted 11 November 2020

A) Check for updates

C Author(s) (or their employer(s)) 2020. Re-use permitted under CC BY-NC. No commercial re-use. See rights and permissions. Published by BMJ.

${ }^{1}$ Department of Epidemiology and Health Statistics, School of Public Health, North China University of Science and Technology, Tangshan, Hebei Province, China

${ }^{2}$ Department of Epidemiology and Health Statistics, School of Public Health, Xinxiang Medical University, Xinxiang, Henan Province, China

Correspondence to Professor Juxiang Yuan; yuanjx@ncst.edu.cn

\section{ABSTRACT}

Objective To examine the associations of rotating night shift work with hyperhomocysteinaemia ( $\mathrm{HHcy}$ ) odds by different exposure metrics.

Design Cross-sectional study.

Setting Occupational physical examination centre for steel production workers, Tangshan, China.

Participants A total of 6846 steelworkers, aged 22-60 years, from the baseline survey of a Chinese occupational cohort.

Primary and secondary outcome measures Different exposure metrics of night shift work, including current shift status, duration of night shifts (years), cumulative number of night shifts (nights), cumulative length of night shifts (hours), average frequency of night shifts (nights/month), average length of night shifts (hours/night) and percentage of hours on night shifts, were used to examine the effects of past and current night shift work on HHcy odds. The total homocysteine concentration in the plasma above 15 $\mu \mathrm{mol} / \mathrm{L}$ was defined as HHcy.

Results Compared with those who never worked night shifts, current night shift workers had elevated odds of HHcy (OR 1.23, 95\% Cl 1.06 to 1.44). Considering a person's lifetime work schedule and compared with individuals who never worked night shifts, duration of night shifts $>28$ years (OR 1.35, 95\% $\mathrm{Cl} 1.12$ to 1.61 ), average frequency of night shifts $>7$ nights/month $(0 \mathrm{R}$ $1.25,95 \% \mathrm{Cl} 1.07$ to 1.47$)$ and percentage of hours on night shifts $>30 \%$ (OR $1.23,95 \% \mathrm{Cl} 1.05$ to 1.43 ) were associated with higher HHcy odds. The duration of night shifts $>20$ years and the average frequency of night shifts $>7$ nights/month could significantly increase the odds of $\mathrm{HH}$ cy regardless of whether the average length of night shifts was greater than 8 hours/night. After stratification by sex, no significant association was found in female workers between different exposure metrics of night shift work and HHcy.

Conclusions Long duration and high frequency of night shift work are associated with higher HHcy odds among male steelworkers.

\section{INTRODUCTION}

It has long been recognised that shift workers are at risk of suffering adverse health outcomes due to misalignment between the circadian
Strengths and limitations of this study

The present study highlights the effects of different exposure metrics of night shift work on hyperhomocysteinaemia (HHcy), since complete avoidance of rotating night shift work is difficult due to socioeconomic realities.

- This study included detailed information on shift work by interview and verified by company records, which makes this exposure assessment reliable.

- Few studies have evaluated the impact of night shift work on HHcy among females; this study is an exception.

- This study cannot evaluate the long-term trend of homocysteine based on a one-time measurement.

- Given the cross-sectional nature of this study, we cannot infer the temporality of night shift work and HHcy.

clock and behavioural cycles. The disruption of the circadian rhythm has been implicated in the pathogenesis of many diseases. The discovery of the molecular mechanism of the circadian clock, which has been recognised with the awarding of the Nobel Prize in Physiology and Medicine in 2017, has brought the study of circadian rhythm and its health consequences to a new era. ${ }^{1}$ Moreover, the International Agency for Research on Cancer has classified night shift work involving circadian disruption as 'probably carcinogenic to humans' (group 2A) according to the latest evaluation of the carcinogenicity in $2019 .^{2}$ Despite the adverse effects of night shift work, our society is increasingly dependent on round-the-clock production and activities to coordinate with the socioeconomic realities. Approximately $20 \%$ of workers worldwide are engaged in night shift work. ${ }^{3}$ In this circumstance, interpreting the effects of different exposures of night shift work can provide evidence for health-related interventions 
among night shift workers, since complete avoidance of rotating night shift work is difficult.

Hyperhomocysteinaemia (HHcy) is a medical condition characterised by an abnormally high levels of total homocysteine (tHcy) in the plasma (tHcy concentrations $>15 \mu \mathrm{mol} / \mathrm{L})$. The pooled prevalence of HHcy was $27.5 \%$ in China. ${ }^{4}$ HHcy has been claimed to be a significant risk factor for a number of disease states, including cardiovascular disease (CVD) ${ }^{5}$ neuropsychiatric illness ${ }^{67}$ and fractures. ${ }^{8}$ A previous meta-analysis showed that shift work was associated with myocardial infarction, ischaemic stroke and coronary events without considering the duration of shift work. ${ }^{9}$ In addition, a subsequent study updated the evidence on the association between shift work and CVD mortality and morbidity risk, which even concluded that there was a nonlinear dose-response relationship between duration of shift work and CVD risk. ${ }^{10}$

Rhythmicity in homocysteine levels has been observed in a previous study. ${ }^{11}$ The misalignment due to night shift work may be implicated in the pathogenesis of HHcy by disrupting glucose metabolism. ${ }^{12}$ Additionally, desynchronisation between circadian clocks and the external world light/dark cycle due to night-time light exposure could contribute to the suppression of melatonin secretion, which in turn leads to HHcy. ${ }^{13}{ }^{14}$ Shift work and HHcy as risk factors for CVD have been supported by a large number of epidemiological studies, but whether shift work-induced disruption of the circadian rhythm also contributes to HHcy remains to be determined.

Previous studies have shown an association between night shift work and HHcy. ${ }^{15}{ }^{16}$ However, it must be noted that there is considerable variability in the detail, quality and definitions of exposure information on night shift work, since implementation of night shift work differs across regions and employment sectors. In addition, considering that reducing homocysteine levels does not improve cardiovascular outcomes and since avoiding night shifts is unrealistic, ${ }^{17}$ it is, therefore, necessary to explore the effect of different night shift work exposures on HHcy for the formulation of effective preventive measures. To the best of our knowledge, there is currently no study on the relationship between the duration, frequency, and intensity of night shift work and HHcy. In this study, different exposure metrics including current shift status, duration of night shifts (years), cumulative number of night shifts (nights), cumulative length of night shifts (hours), average frequency of night shifts (nights/month), average length of night shifts (hours/ night) and percentage of hours on night shifts, were used to examine the effects of past and current night shift work on HHcy odds.

\section{METHODS}

\section{Study design and population}

This study reported results from the baseline survey of a Chinese occupational cohort conducted among steelworkers who were prospectively recruited at 11 steel

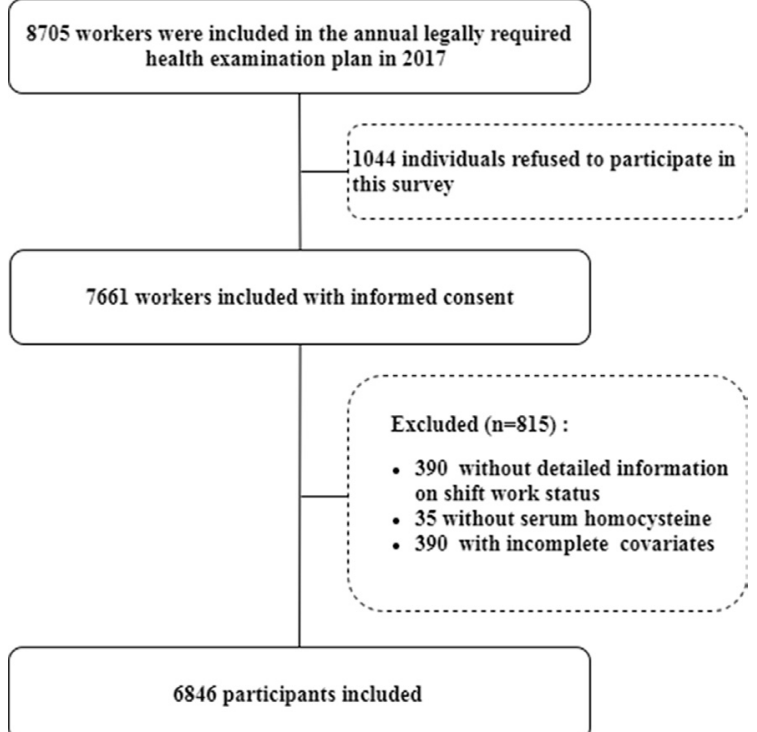

Figure 1 Flow chart of the participant selection.

production departments owned by the HBIS Group's Tangsteel Company in Tangshan City, Hebei Province in north China. ${ }^{18}$ All workers at this company underwent a legally required health examination each year. From February to June 2017, 7661 participants were recruited. Dust, heat stress, noise and carbon monoxide are the major occupational hazards to the current workers. Workers without detailed lifetime shift work information, those who provided incomplete covariate data on the questionnaire, and those who did not have homocysteine levels in the plasma were excluded; 6846 participants were included in this study (figure 1). Compared with workers excluded, those who were included were older $(44.2 \pm 8.0$ years vs $34.4 \pm 7.7$ years, $\mathrm{p}<0.001)$, and had a higher proportion of women $(8.54 \%$ vs $5.90 \%, \mathrm{p}=0.010)$. All participants gave informed consent before taking part in this study.

\section{Ascertainment of HHcy}

Fasting blood was drawn from the participant's forearm vein between 08:00 and 09:30 hours. For night shift workers, blood was drawn in the morning of a day off work. The enzymatic cycling method was used to test the homocysteine concentration in the plasma (HCY kit, Beijing Strong Biotechnologies, Beijing, China). Withinlaboratory intra-assay and interassay variable coefficients for serum homocysteine were $<5 \%$ and $<9 \%$, respectively. tHcy concentration in the plasma above $15 \mu \mathrm{mol} / \mathrm{L}$ was defined as HHcy. ${ }^{19}$

\section{Assessment of night shift work}

The main work schedule of the present study population has been introduced in detail in our previous research. ${ }^{18}$ In brief, shift work in this study refers to rotating night shifts (the mainly four-crew-three-shift system now and historical three-crew-two-shift system). Workers who worked regular working hours at all times were defined as never night workers. In this study, the detailed lifetime 
employment history was collected by face-to-face personal interviews and all the reported information was verified with the company's records. Participants who were recruited were asked to report whether they were involved in rotating night shift work (working through 00:00 to 6:00 hours) during their employment (current shift status: 'never', 'ever, 'current'). If yes (ever or current), they would be further asked about the start and end dates of each shift system, the average number of night shifts per month in each shift system, the daily working hours, and usual days off per month. Using that information, different exposure metrics of night shift were derived. Duration of night shifts (years): sum of years spent in all different night shift systems; cumulative number of night shifts (nights): sum of nights spent in all different night shift systems; cumulative length of night shifts (hours): sum of hours spent in all different night shift systems; average length of night shifts (hours/night): cumulative length of night shifts (hours) divided by cumulative number of night shifts (nights); average frequency of night shifts (nights/month): cumulative number of night shifts (nights) divided by cumulative number of months of employment; percentage of hours on night shifts: cumulative length of night shifts (hours) divided by total working hours of employment. ${ }^{20} 21$

\section{Assessment of covariates}

Covariates mainly included age, sex, body mass index (BMI), smoking status, drinking status, educational level, physical activity, sedentary behaviour, sleep duration, insomnia, diet (dietary approaches to stop hypertension, DASH), diabetes, dyslipidaemia, hypertension, liver enzyme abnormality and decreased estimated glomerular filtration rate (eGFR) (see online supplemental appendix).

\section{Statistical analysis}

Continuous variables are presented as the means and $\mathrm{SD}$, and between-group comparisons were performed using Student's t-test if the data were normally distributed. Otherwise, the median (upper quartile-lower quartile) and Wilcoxon Scores (rank sums) test were used to describe and compare these continuous variables between groups. Categorical variables are presented as numbers and percentages, and the $\chi^{2}$ test was used to compare differences among groups.

Multiple logistic regression models were used to examine the association of different exposure metrics of night shift work in relation to HHcy prevalence. Potential confounders, including age, sex, BMI, smoking status, dyslipidaemia, hypertension and decreased eGFR, were included in the multiple analysis. For continuous exposure variables, restricted cubic spline (RCS) models were also used to visually examine the association between night shift work and tHcy (as continuous and categorical variables, respectively) with adjustment for potential confounders. For the independent effects and interactions of duration, frequency and intensity of night shifts on HHcy, the medians of duration of night shifts, average frequency of night shifts and average length of night shifts were chosen as cut-off points to secure a reasonable number of observations in each exposure category and cross-classification group.

We tested for the presence of multiplicative interaction by including a cross-product term between the duration of night shifts and the average frequency of night shifts in our fully adjusted multivariable model. To assess additive interaction between duration of night shifts and average frequency of night shifts on odds of HHcy, indicators including relative excess risk due to interaction and attributable proportion due to interaction were used. ${ }^{22}$ $\mathrm{p}<0.05$ was regarded as significant for two-sided tests. Several sensitivity analyses were performed to test the robustness of our results, including further adjustments for major occupational hazards, and the elimination of the last $1 \%$ quantile of the duration of night shifts. All statistical analyses were performed using SAS V.9.3 (SAS Institute).

\section{Patient and public involvement}

No patients were involved in the development of the research question and outcome measures, design, recruitment to and conduct of the study. The study results will be disseminated to participants by our customised app.

\section{RESULTS \\ General characteristics of the participants}

Table 1 shows the general characteristics of study participants according to HHcy status. The present study of 6846 included participants consisted of $91.5 \%$ males, with a mean age of 44.2 years. Among all workers included, the proportions of ever and current night shift workers were $21.8 \%$ and $63.2 \%$, respectively. Overall, workers with HHcy were more likely to be current night shift workers, men, current smokers and those with hypertension or hyperlipidaemia. As shown in online supplemental table $\mathrm{S} 1$, the prevalence rates of HHcy in males and females were $32.4 \%$ and $10.8 \%$, respectively. Online supplemental table S2 shows the basic characteristics of the participants according to the duration of night shifts. Current smoking, current drinking and sedentary behaviour were more likely to be reported among long-term shift workers. In terms of current health status, long-term shift workers also showed higher prevalence rates of diabetes, dyslipidaemia, hypertension and a lower eGFR. In addition, the prevalence of HHcy also showed age difference (online supplemental table S3).

\section{Different exposure metrics of night shift work and HHcy}

The prevalence rates of HHcy among ever night shift workers and current night shift workers were $28.9 \%$ and $31.9 \%$, respectively, which were higher than the rate among those who never worked night shifts $(27.3 \%)$. After classification by different exposure metrics of night shift work, higher HHcy prevalence rates were observed 
Table 1 Basic characteristics according to HHcy status

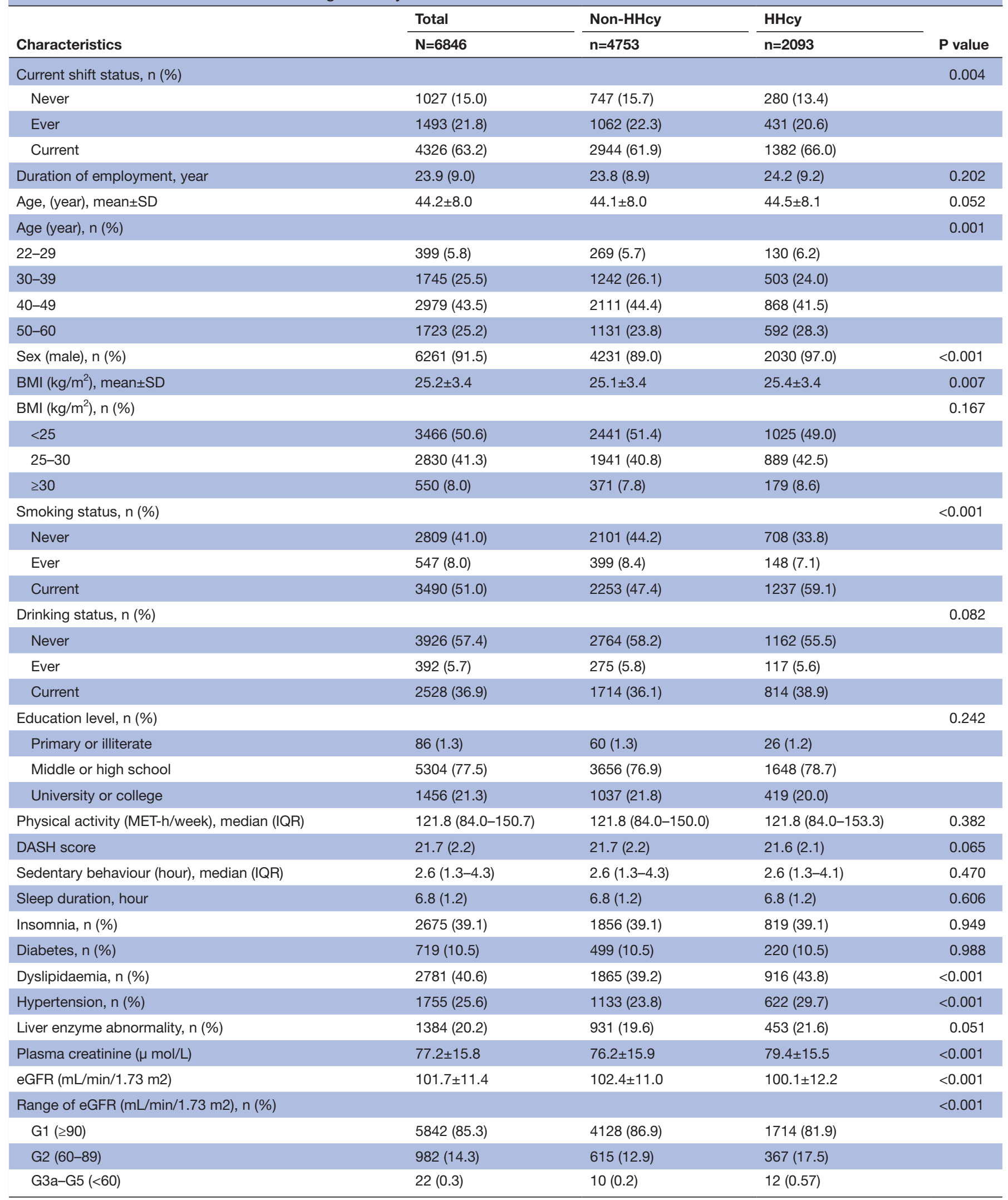

Values are expressed as the mean \pm SD or median (IQR) or number (\%); $p$ values were from Pearson's $\chi^{2}$ test for categorical variables and Student's $\mathrm{t}$-test or Wilcoxon scores (rank sums) for continuous variables.

BMI, body mass index; DASH, dietary approaches to stop hypertension; eGFR, estimated glomerular filtration rate; HHcy, hyperhomocysteinaemia; MET, metabolic equivalent units. 
in the groups with higher exposure categories of night shift work (online supplemental figure S1). As shown in table 2, compared with those who never worked night shifts, significantly elevated odds of HHcy were observed in the highest exposure categories (model 1). After adjustment for age and sex, these estimates were attenuated but remained significant (model 2). Model 3 additionally adjusted for BMI, smoking status, drinking status, dyslipidaemia, hypertension and decreased eGFR, and the results were overall comparable to Model 2. In the multivariable adjustment model (Model 3), current shift workers had higher HHcy odds than those who never worked night shifts (OR 1.23, 95\% CI 1.06 to 1.44 ). Participants in the highest exposure categories of duration of night shifts, cumulative number of night shifts, cumulative length of night shifts, average frequency of night shifts, average length of night shifts and percentage of hours on night shifts had increased odds of HHcy, with ORs $(95 \% \mathrm{CI})$ of 1.35 (1.12 to 1.61$), 1.34$ (1.11 to 1.61 ), 1.33 (1.11 to 1.61 ), 1.25 (1.07 to 1.47 ), 1.27 (1.01 to 1.60 ) and 1.23 (1.05 to 1.43 ), respectively, when compared with those who never worked night shifts (table 2, model 3 ). The odds of HHcy associated with other categories among different metrics of night shift work and their linear trends can also be observed in online supplemental figure S2. In the RCS models, positive correlations between homocysteine (as a continuous variable: plasma tHcy concentration $(\mu \mathrm{mol} / \mathrm{L})$, or a binary variable: HHcy (yes/no)) and duration of night shifts (continuous, years), and cumulative number of night shifts (continuous, nights), cumulative length of night shifts (continuous, hours) were observed (figure 2).

We then evaluated the independent effects of current shift status, duration of night shifts (years), average frequency of night shifts (nights/month) and average length of night shifts (hours/night) in relation to HHcy among night shift workers (ever or current). After mutually adjusting for current shift status duration of night shifts (years), average frequency of night shifts (nights/ month), average length of night shifts (hours/night) and other confounders, the highest levels of exposure to night shift work independently increased the odds of HHcy, but this increase was insignificant (online supplemental table S4). Cross-classification results showed that exposure to duration of night shifts $>20$ years and average frequency of night shifts $>7$ nights/month can significantly increase the odds of HHcy (table 3). The duration of night shifts $>20$ years and the average frequency of night shifts $>7$ nights/month were jointly associated with higher odds of HHcy (OR 1.20, 95\% CI 1.03 to 1.39). However, no significant multiplicative or additive interactions between the duration of night shifts and the average frequency of night shifts on the odds of HHcy were observed (online supplemental table S5).

The results of stratified analysis by sex showed that the association between different exposure metrics of night shift work and HHcy among male workers was comparable to the total study population. However, no significant associations between different exposure categories of night shift work and HHcy were observed among female workers (online supplemental table S6).

\section{Sensitivity analyses}

Considering that dust, heat stress, noise and carbon monoxide are the major occupational hazards to the current steelworkers, we additionally adjusted for these exposures on the basis of model 3 in table 2, and the results were similar to model 3 in table 2 (online supplemental table S7). Moreover, to avoid the influence of the maximum value on the fitting result of RCSs, we removed the last $1 \%$ quantile of the duration of night shifts (years), the cumulative number of night shifts (nights) and the cumulative length of night shifts (hours), and the relationships remained robust (online supplemental figure S3).

\section{DISCUSSION}

This study examined the cross-sectional association of night shift work with HHcy odds among 6846 steelworkers, and found that elevated odds of HHcy were associated with the highest exposure categories of night shift work when applying different exposure metrics for night shift work. The present study also provided additional evidence concerning dose-response relationships between the duration of night shifts and HHcy among steelworkers, which have never been reported in previous studies. Moreover, long duration of night shifts $(>20$ years) and high frequency of night shifts ( $>7$ nights/ month) were jointly associated with higher odds of HHcy.

Consistent with our findings, several previous studies have found that shift work is related to higher levels of plasma homocysteine. ${ }^{151623}$ In addition, what we already know is that HHcy is an independent risk factor for CVD, and largely confirms epidemiological data showing that night shift workers are at increased risk of CVD,${ }^{24}$ which indirectly supports the association between night shift work and HHcy. It is noteworthy that the previous studies on the relationship between night shift work and HHcy often have a coarse assessment of night shift work (usually divided into two categories: day workers and shift workers). However, simply exploring the relationship between night shift work (yes/no) and HHcy is not enough to provide guideline recommendations regarding the risk related to shift schedules, since complete avoidance of rotating night shift work is difficult when socioeconomic realities are taken into consideration. Our findings highlight the effect of different exposure metrics of night shift work on HHcy and extend the positive association between night shift work and odds of HHcy. In addition to the duration of night shifts, we also found that the frequency and length of night shifts are related to odds of HHcy. A high shift rotation frequency increases the likelihood of 'quick returns' between shifts, ${ }^{25}$ which can serve as a 'big problem in life' among some shift workers. ${ }^{26}$ In previous studies, high shift rotation frequency has been associated 
Table 2 Associations of different exposure metrics of night shift work with HHcy odds

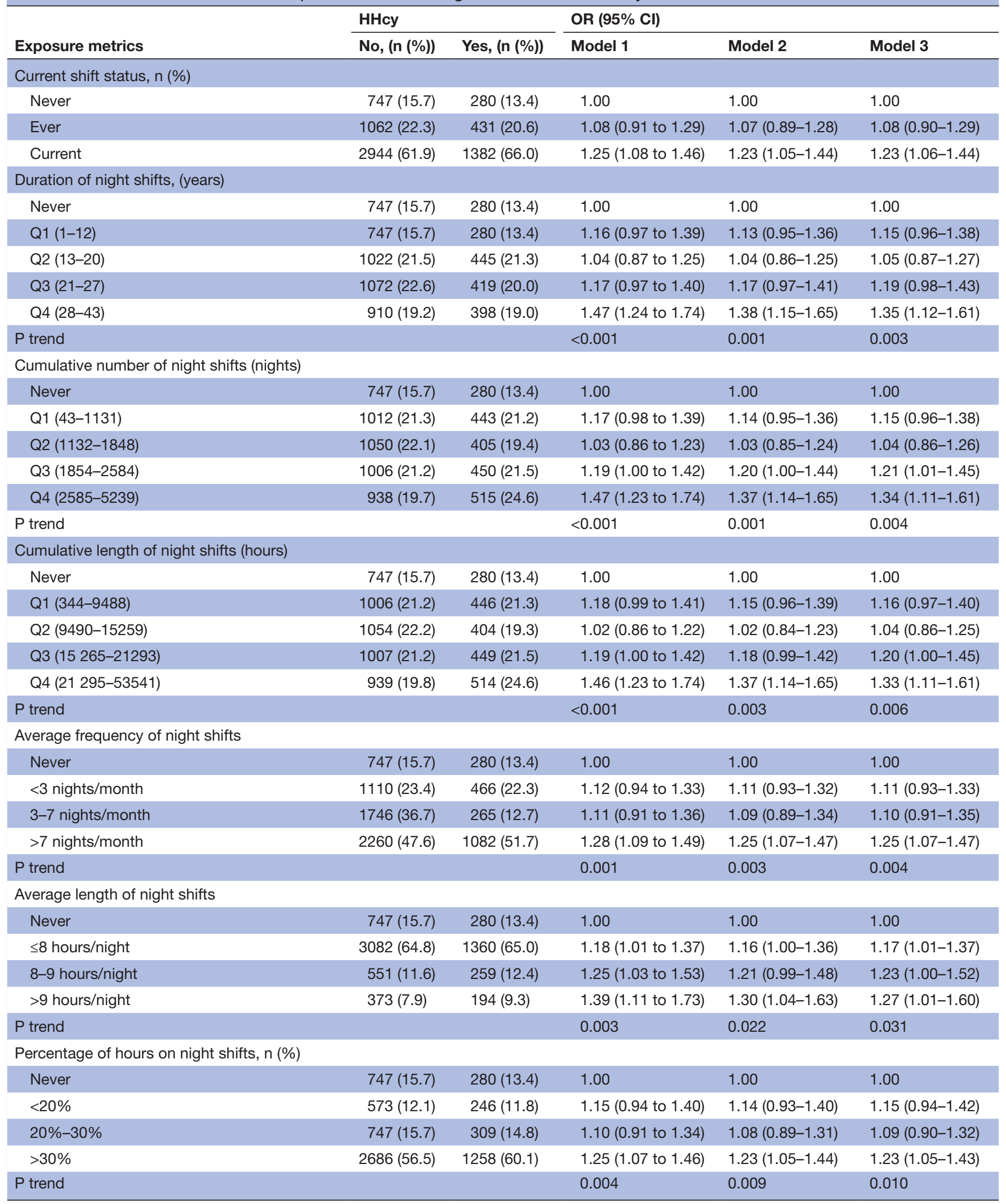

Model 1: unadjusted; model 2: adjusted for age and sex; model 3: adjusted for age, sex, BMI, smoking status, dyslipidaemia, hypertension and decreased eGFR. The cut-off points of average frequency of night shifts, average length of night shifts and percentage of hours on night shifts were chosen to secure a reasonable number of observations in each category.

BMI, body mass index; eGFR, estimated glomerular filtration rate; HHcy, hyperhomocysteinaemia.; 

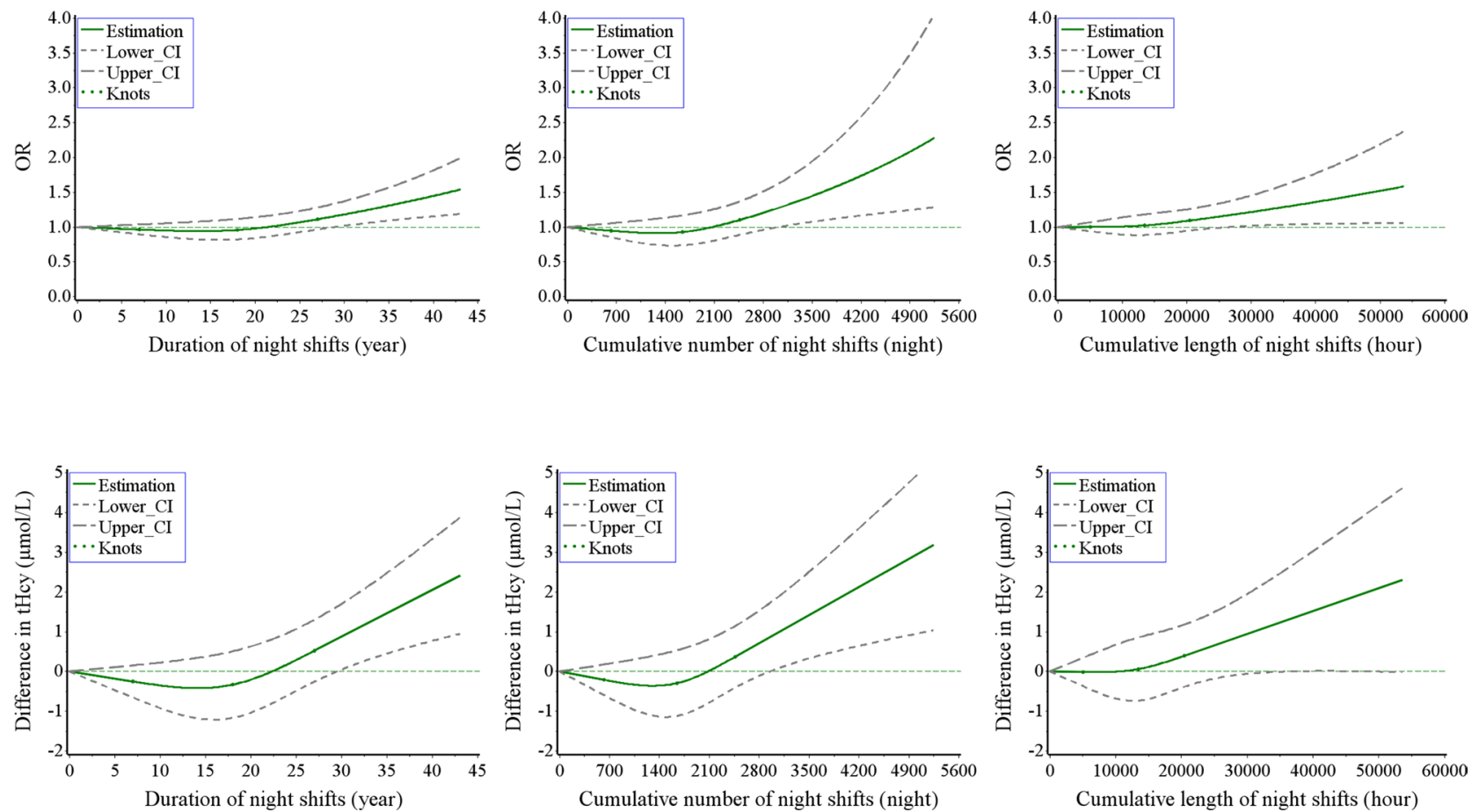

Figure 2 Associations of duration, cumulative number and cumulative length of night shifts with thcy (as a continuous or a binary variable) from restricted cubic spline models. 'Difference in tHcy' indicates difference of thcy ( $\mu \mathrm{mol} / \mathrm{L})$ levels in the plasma where the reference values for duration, cumulative number and cumulative length of night shifts are all 0 (never worked night shifts). Adjusted for age, sex, BMI, smoking status, dyslipidaemia, hypertension and decreased eGFR. BMI, body mass index; eGFR, estimated glomerular filtration rate; HHcy, hyperhomocysteinaemia; tHcy, total homocysteine.

with employee performance and health, including poor sleep quality, disrupted social relationships ${ }^{27}$ and type 2 diabetes. ${ }^{28}$ In terms of shift length (between 8 and 12 hours shifts), the effect of long shifts on employee performance and satisfaction has been widely explored in occupational sectors, with inconsistent results. ${ }^{29}$ Based on the results of previous large cross-sectional studies conducted among nurses (sample size ranging from 3710 to 22275 nurses) in the USA that concluded that long shifts were negatively associated with employees' job performance and psychological well-being, ${ }^{30-32}$ our study expanded the evidence of adverse effects of long shifts on physical health. The added finding in this study is that when the duration, frequency, and length of night shifts are considered simultaneously, significantly higher odds of HHcy are limited to participants who have been exposed to duration of night shifts $>20$ years and have a frequency of night shifts $>7$ nights $/$ month. This implies that for long duration night shift workers, modifying the rotation frequency of night shifts (ie, $<7$ nights /

Table 3 Association between night shift work and HHcy odds by cross-classification analysis

\begin{tabular}{llllll}
\hline $\begin{array}{l}\text { Duration of } \\
\text { night shifts }\end{array}$ & $\begin{array}{l}\text { Average frequency of } \\
\text { night shifts }\end{array}$ & $\begin{array}{l}\text { Average length of } \\
\text { night shifts }\end{array}$ & HHcy & No, (n (\%)) & Yes, (n (\%)) \\
\cline { 4 - 5 } OR (95\% Cl)
\end{tabular}

Adjusted for age, sex, BMI, smoking status, dyslipidaemia, hypertension and decreased eGFR. The cut-off points of the duration of night shifts, average frequency of night shifts and average length of night shifts were the median of the corresponding continuous variables. $\mathrm{BMI}$, body mass index; eGFR, estimated glomerular filtration rate; HHcy, hyperhomocysteinaemia.; 
month) may reduce the odds of HHcy. Moreover, given that both exposure and outcome may be related to sex, we added female-specific findings. However, no association was observed between night shift work and HHcy in females. Considering the relationship between HHcy and CVD, our finding was indirectly supported by a previous study, which reported that shift work was associated with carotid atherosclerosis only in men, but not in women. ${ }^{33}$ In fact, due to sex differences in fat-free mass and estradiol concentrations, the level of plasma tHcy in males is consistently higher than that in females in the general population. ${ }^{34}$ This could explain the sex discrepancies, at least in part, in the effect of night shift work on HHcy. Considering the small sample size of women in this study, the lack of association between night shift work and HHcy in women should be verified in large-scale cohort studies.

Several possible pathways from night shift work to negative health outcomes have been established, such as circadian misalignment, shift work-related unhealthy lifestyles and stress due to a disturbed social life. Numerous physiological functions exhibit substantial circadian oscillations synchronised with the sleep/wake and light/dark cycles. Rhythmicity in homocysteine levels has been observed in a previous study. ${ }^{11}$ The misalignment between the circadian clock and behavioural cycles, including rest/activity and feeding/fasting cycles due to night shift work, may be implicated in the pathogenesis of HHcy by disrupting glucose metabolism. ${ }^{12} 35$ Additionally, previous evidence has shown that under conditions where melatonin levels are decreased, there would be a possible increase in tHcy concentrations. ${ }^{13}$ Therefore, desynchronisation between circadian clocks and the external world light/dark cycle due to nighttime light exposure could contribute to the suppression of melatonin secretion, ${ }^{14}$ which in turn leads to HHcy. Moreover, shift workers usually had poorer health habits than day workers, including smoking and unhealthy diet, ${ }^{33}$ which were strongly associated with HHcy. ${ }^{36}{ }^{37}$ Furthermore, chronic stress due to a disturbed social life may also explain the elevated risk of HHcy in shift workers. ${ }^{38} 39$

The major strengths of our study include detailed shift work information, lifestyle information and health status related to both exposure and outcome, and a large sample size. To our knowledge, this is the first study to explore the relationship between different exposure metrics of night shifts and HHcy. Despite the limited sample size, we also conducted an exploratory sex-specific analysis of the relationship between night shift work and odds of HHcy. The present study also has certain limitations. First, given the cross-sectional nature of this study, we cannot infer the temporality of night shift work and HHcy, nor can we evaluate the long-term trend of tHcy based on a one-time measurement. Second, plasma levels of vitamin $\mathrm{B}_{6}$, folic acid (vitamin $\mathrm{B}_{9}$ ) and vitamin $\mathrm{B}_{12}$ were not tested, deficiencies of which can directly lead to an increase in homocysteine levels. ${ }^{40}$ Nonetheless, this may not be regarded as a major bias, since we considered the distribution of the DASH diet (rich in fruits, vegetables, whole grains and dairy), which has beneficial effects on homocysteine that have been confirmed in previous studies ${ }^{41}$ Third, genetic defects (such as polymorphisms at MTHFR C677T and MTR A2756G) in 5-MTHF reductase can consequently lead to HHcy, ${ }^{42}{ }^{43}$ but due to funding constraints we did not analyse the genotypes of the relevant sites of the participants. However, a previous well-designed case-control study showed that higher levels of plasma tHcy in shift-working bus drivers were not secondary to other biochemical problems, including folic acid, vitamin $\mathrm{B}_{12}$ and $\mathrm{C} 677 \mathrm{~T}$ mutations, ${ }^{15}$ meaning that our results still have a certain reference value. Fourth, the absence of chronotype information may have led to a confounding bias. Finally, this study was conducted in the steel production occupational setting, which limits the results of this study to the general population.

\section{CONCLUSIONS}

In conclusion, this study showed that when the duration, frequency and length of night shifts are considered simultaneously, significantly higher odds of HHcy are limited to participants with a long duration of night shifts $(>20$ years) combined with a high frequency of night shifts (>7 nights/month). These findings imply that for longduration night shift workers, modifying the rotation frequency of night shifts may reduce the odds of HHcy. Further prospective studies are warranted to confirm our findings.

Acknowledgements We thank all members involving the collection of baseline data.

Contributors SZ raised the study concept and drafted the manuscript. JY designed this work. YW, ZW and HW analysed the data. CX, QL and YZ provided inputs and revisions. JY and WG supervised the fieldwork of this project. All authors agreed to submit this article.

Funding This work was supported by the National Key R\&D Program of China (No.2016YFC0900605).

Competing interests None declared.

Patient consent for publication Obtained.

Ethics approval This research was approved by the Ethics Committee of North China University of Science and Technology (No.15006).

Provenance and peer review Not commissioned; externally peer reviewed.

Data availability statement Data may be obtained from a third party and are not publicly available.

Supplemental material This content has been supplied by the author(s). It has not been vetted by BMJ Publishing Group Limited (BMJ) and may not have been peer-reviewed. Any opinions or recommendations discussed are solely those of the author(s) and are not endorsed by BMJ. BMJ disclaims all liability and responsibility arising from any reliance placed on the content. Where the content includes any translated material, BMJ does not warrant the accuracy and reliability of the translations (including but not limited to local regulations, clinical guidelines, terminology, drug names and drug dosages), and is not responsible for any error and/or omissions arising from translation and adaptation or otherwise.

Open access This is an open access article distributed in accordance with the Creative Commons Attribution Non Commercial (CC BY-NC 4.0) license, which permits others to distribute, remix, adapt, build upon this work non-commercially, and license their derivative works on different terms, provided the original work is properly cited, appropriate credit is given, any changes made indicated, and the use is non-commercial. See: http://creativecommons.org/licenses/by-nc/4.0/. 


\section{ORCID iDs}

Shengkui Zhang http://orcid.org/0000-0003-0859-6884

Ying Zhu http://orcid.org/0000-0003-0949-0475

\section{REFERENCES}

1 Callaway E, Ledford H. Medicine Nobel awarded for work on circadian clocks. Nature 2017;550:18.

2 IARC Monographs Vol 124 group. Carcinogenicity of night shift work. Lancet Oncol 2019;20:1058-9.

3 De Bacquer D, Van Risseghem M, Clays E, et al. Rotating shift work and the metabolic syndrome: a prospective study. Int $J$ Epidemiol 2009;38:848-54.

4 Yang B, Fan S, Zhi X, et al. Prevalence of hyperhomocysteinemia in China: a systematic review and meta-analysis. Nutrients 2014;7:74-90.

5 Cattaneo M. Hyperhomocysteinemia, atherosclerosis and thrombosis. Thromb Haemost 1999;81:165-76.

6 Morris MS. Homocysteine and Alzheimer's disease. Lancet Neurol 2003;2:425-8.

7 Dietrich-Muszalska A, Malinowska J, Olas B, et al. The oxidative stress may be induced by the elevated homocysteine in schizophrenic patients. Neurochem Res 2012;37:1057-62.

8 van Meurs JBJ, Dhonukshe-Rutten RAM, Pluijm SMF, et al. Homocysteine levels and the risk of osteoporotic fracture. $N$ Engl J Med 2004;350:2033-41.

9 Vyas MV, Garg AX, lansavichus AV, et al. Shift work and vascular events: systematic review and meta-analysis. BMJ 2012;345:e4800.

10 Torquati L, Mielke GI, Brown WJ, et al. Shift work and the risk of cardiovascular disease. A systematic review and meta-analysis including dose-response relationship. Scand J Work Environ Health 2018;44:229-38.

11 Paul B, Saradalekshmi KR, Alex AM, et al. Circadian rhythm of homocysteine is hCLOCK genotype dependent. Mol Biol Rep 2014;41:3597-602.

12 Henderson DC, Copeland PM, Nguyen DD, et al. Homocysteine levels and glucose metabolism in non-obese, non-diabetic chronic schizophrenia. Acta Psychiatr Scand 2006;113:121-5.

13 Baydas G, Gursu MF, Cikim G, et al. Homocysteine levels are increased due to lack of melatonin in pinealectomized rats: is there a link between melatonin and homocysteine? J Pineal Res 2002;32:63-4.

14 Touitou Y, Reinberg A, Touitou D. Association between light at night, melatonin secretion, sleep deprivation, and the internal clock: health impacts and mechanisms of circadian disruption. Life Sci 2017;173:94-106.

15 Martins PJF, D'Almeida V, Vergani N, et al. Increased plasma homocysteine levels in shift working bus drivers. Occup Environ Med 2003;60:662-6.

16 Kang D, Kang S-K, Choi W-J, et al. Association between shift work and hyperhomocysteinemia in male workers. Ann Occup Environ Med 2019;31:e1.

17 Martí-Carvajal AJ, Solà I, Lathyris D, et al. Homocysteine-Lowering interventions for preventing cardiovascular events. Cochrane Database Syst Rev 2017;8:Cd006612.

18 Zhang S, Wang Y, Wang Z, et al. Rotating night shift work and nonalcoholic fatty liver disease among steelworkers in China: a crosssectional survey. Occup Environ Med 2020;77:333-9.

19 Guo H, Chi J, Xing Y, et al. Influence of folic acid on plasma homocysteine levels \& arterial endothelial function in patients with unstable angina. Indian J Med Res 2009;129:279-84.

20 Charles LE, Gu JK, Fekedulegn D, et al. Association between shiftwork and glomerular filtration rate in police officers. J Occup Environ Med 2013;55:1323-8.
21 Lie J-AS, Kjuus H, Zienolddiny S, et al. Night work and breast cancer risk among Norwegian nurses: assessment by different exposure metrics. Am J Epidemiol 2011;173:1272-9.

22 Andersson T, Alfredsson L, Källberg H, et al. Calculating measures of biological interaction. Eur J Epidemiol 2005;20:575-9.

23 Lavie L, Lavie P. Elevated plasma homocysteine in older shiftworkers: a potential risk factor for cardiovascular morbidity. Chronobiol Int 2007;24:115-28.

24 Wang D, Ruan W, Chen Z, et al. Shift work and risk of cardiovascular disease morbidity and mortality: a doseresponse meta-analysis of cohort studies. Eur J Prev Cardiol 2018;25:1293-302.

25 Hall AL, Franche R-L, Koehoorn M. Examining exposure assessment in shift work research: a study on depression among nurses. Ann Work Expo Health 2018;62:182-94.

26 Åkerstedt T, Kecklund G. What work schedule characteristics constitute a problem to the individual? A representative study of Swedish shift workers. Appl Ergon 2017;59:320-5.

27 Vedaa Øystein, Harris A, Bjorvatn B, et al. Systematic review of the relationship between quick returns in rotating shift work and healthrelated outcomes. Ergonomics 2016;59:1-14.

28 Vetter C, Dashti HS, Lane JM, et al. Night shift work, genetic risk, and type 2 diabetes in the UK Biobank. Diabetes Care 2018;41:762-9.

29 Dall'Ora C, Ball J, Recio-Saucedo A, et al. Characteristics of shift work and their impact on employee performance and wellbeing: a literature review. Int J Nurs Stud 2016;57:12-27.

30 Stimpfel AW, Sloane DM, Aiken LH. The longer the shifts for hospital nurses, the higher the levels of burnout and patient dissatisfaction. Health Aff 2012;31:2501-9.

31 Stimpfel AW, Lake ET, Barton S, et al. How differing shift lengths relate to quality outcomes in pediatrics. J Nurs Adm 2013;43:95-100.

32 Stimpfel AW, Aiken LH. Hospital staff nurses' shift length associated with safety and quality of care. J Nurs Care Qual 2013;28:122-9.

33 Puttonen S, Kivimäki M, Elovainio M, et al. Shift work in young adults and carotid artery intima-media thickness: the cardiovascular risk in young Finns study. Atherosclerosis 2009;205:608-13.

34 Aparicio-Ugarriza R, Palacios G, Alder M, et al. A review of the cutoff points for the diagnosis of vitamin B12 deficiency in the general population. Clin Chem Lab Med 2015;53:1149-59.

35 Spiegel K, Leproult R, Van Cauter E. Impact of sleep debt on metabolic and endocrine function. Lancet 1999;354:1435-9.

36 Loprinzi PD, Branscum A, Hanks J, et al. Healthy lifestyle characteristics and their joint association with cardiovascular disease biomarkers in US adults. Mayo Clin Proc 2016;91:432-42.

37 Chen S, Guo X, Dong S, et al. Relationship between lifestyle factors and hyperhomocysteinemia in general Chinese population: a crosssectional study. Postgrad Med 2017;129:216-23.

38 Härmä M. Workhours in relation to work stress, recovery and health. Scand J Work Environ Health 2006;32:502-14.

39 Jendricko T, Vidović A, Grubisić-llić M, et al. Homocysteine and serum lipids concentration in male war veterans with posttraumatic stress disorder. Prog Neuropsychopharmacol Biol Psychiatry 2009;33:134-40.

40 Miller JW, Nadeau MR, Smith D, et al. Vitamin B-6 deficiency vs folate deficiency: comparison of responses to methionine loading in rats. Am J Clin Nutr 1994;59:1033-9.

41 Appel LJ, Miller ER, Jee SH, et al. Effect of dietary patterns on serum homocysteine: results of a randomized, controlled feeding study. Circulation 2000;102:852-7.

42 Qin X, Li J, Cui Y, et al. Mthfr C677T and mtr A2756G polymorphisms and the homocysteine lowering efficacy of different doses of folic acid in hypertensive Chinese adults. Nutr J 2012;11:2.

43 Yakub M, Moti N, Parveen S, et al. Polymorphisms in MTHFR, MS and CBS genes and homocysteine levels in a Pakistani population. PLoS One 2012;7:e33222. 